\title{
A FUNCTIONAL RELATION FOR TORNHEIM'S DOUBLE ZETA FUNCTIONS
}

\author{
KAZUHIRO ONODERA
}

\begin{abstract}
In this paper, we generalize the partial fraction decomposition which is fundamental in the theory of multiple zeta values, and prove a relation between Tornheim's double zeta functions of three complex variables. As applications, we give new integral representations of several zeta functions, an extension of the parity result to the whole domain of convergence, concrete expressions of Tornheim's double zeta function at non-positive integers and some results for the behavior of a certain Witten's zeta function at each integer. As an appendix, we show a functional equation for Euler's double zeta function.
\end{abstract}

\section{INTRODUCTION}

Tornheim's double zeta function is defined as

$$
\zeta(s, t ; u)=\sum_{m, n=1}^{\infty} \frac{1}{m^{s} n^{t}(m+n)^{u}}
$$

for $(s, t, u) \in \mathbb{C}^{3}$ with $\operatorname{Re}(s+u)>1, \operatorname{Re}(t+u)>1$ and $\operatorname{Re}(s+t+u)>2$. It is known, by Matsumoto [7, Theorem 1], that $\zeta(s, t ; u)$ can be meromorphically continued to the whole space $\mathbb{C}^{3}$, and its singularities are located on the subsets of $\mathbb{C}^{3}$ defined by one of the equations $s+u=1-l, t+u=1-l(l=0,1,2, \ldots)$ and $s+t+u=2$. This function can be regarded as a generalization of some well-known zeta functions: the product of two Riemann zeta functions $\zeta(s) \zeta(t)=\zeta(s, t ; 0)$, the Euler double zeta function $\zeta(u, t)=\zeta(0, t ; u)$ and the $S U(3)$-type Witten zeta function $\zeta_{S U(3)}(s)=2^{s} \zeta(s, s ; s)$. Euler and Tornheim [12], and many people gave a lot of relations between the values $\zeta(s, t ; u)$ for triples $(s, t, u)$ of non-negative integers on the domain of convergence, but little relation as functions of complex variables has been found. As an exception, Tsumura [13, Theorem 4.5] represented explicitly the function

$$
Z(s, t ; u)=\zeta(s, t ; u)+\cos (\pi t) \zeta(t, u ; s)+\cos (\pi s) \zeta(u, s ; t)
$$

in terms of the Riemann zeta function, when $s, t \in \mathbb{Z}_{\geq 0}, t \geq 2$ and $u \in \mathbb{C}$, except for singularities. Afterward Nakamura [9, Theorem 1.2] gave a simpler version: for $s, t \in \mathbb{Z}_{\geq 1}$ and $u \in \mathbb{C}$

2010 Mathematics Subject Classification. 11M32.

Key words and phrases. double zeta function, Witten zeta function, double zeta value, functional relation, functional equation, partial fraction decomposition.

This work was supported by Grant-in-Aid for JSPS Fellows (No. 22008809). 
except for the singular points,

$$
\begin{aligned}
Z(s, t ; u)= & 2 \sum_{h=0}^{[s / 2]}\left(\begin{array}{c}
s+t-2 h-1 \\
s-2 h
\end{array}\right) \zeta(2 h) \zeta(s+t+u-2 h) \\
& +2 \sum_{k=0}^{[t / 2]}\left(\begin{array}{c}
s+t-2 k-1 \\
t-2 k
\end{array}\right) \zeta(2 k) \zeta(s+t+u-2 k),
\end{aligned}
$$

where $[x]$ for $x \in \mathbb{R}$ denotes the greatest integer not exceeding $x$. This result seems really fascinating because it contains most of the known relations between the values $\zeta(s, t ; u)$ for $s, t, u \in \mathbb{Z}_{\geq 1}$ and the Riemann zeta values (see [9, $\left.\S 3\right]$ ). The aim of this paper is to generalize it to a relation between Tornheim's double zeta functions of three complex variables. Our main result is

Theorem. The following relation holds on the whole space $\mathbb{C}^{3}$ except for the singular points of both sides:

$$
Z(s, t ; u)=A(s, t ; u)+A(t, s ; u),
$$

where, for $(s, t, u) \in \mathbb{C}^{3}$ with $s,-t, 1-t-u \neq 0,1,2, \ldots$,

$$
A(s, t ; u)=\frac{\sin (\pi s)}{2 \pi i} \int_{L} \cot \left(\frac{\pi(s-\eta)}{2}\right) \frac{\Gamma(t+\eta) \Gamma(-\eta)}{\Gamma(t)} \zeta(s-\eta) \zeta(t+u+\eta) d \eta .
$$

Here, the contour $L$ is a line from $-i \infty$ to $i \infty$ indented in such a manner as to separate the poles at $\eta=s-2 n,-t-n, 1-t-u(n=0,1,2, \ldots)$ from the poles at $\eta=0,1,2, \ldots$.

Remark 1.1. The singularities of $A(s, t ; u)$ are located only on the subsets of $\mathbb{C}^{3}$ defined by the equations $t+u=1-l(l=0,1,2, \ldots)$. This can be easily seen by shifting the contour $L$ as follows. Let $K$ be a non-negative integer. If $\operatorname{Re}(s)<K+1 / 2,-K-1 / 2<\operatorname{Re}(t)$ and $-K+1 / 2<\operatorname{Re}(t+u)$, then

$$
\begin{aligned}
A(s, t ; u)= & 2 \sum_{k=0}^{K} \frac{(t)_{k}}{k !} \cos ^{2}\left(\frac{\pi(s-k)}{2}\right) \zeta(s-k) \zeta(t+u+k) \\
& +\frac{\sin (\pi s)}{2 \pi i} \int_{L_{K}} \cot \left(\frac{\pi(s-\eta)}{2}\right) \frac{\Gamma(t+\eta) \Gamma(-\eta)}{\Gamma(t)} \zeta(s-\eta) \zeta(t+u+\eta) d \eta,
\end{aligned}
$$

where $(t)_{k}=\Gamma(t+k) / \Gamma(t)$ and $L_{K}$ describes the vertical line from $K+1 / 2-i \infty$ to $K+1 / 2+i \infty$. Also, from this, it is clear that our functional relation is a generalization of (1.2) (see (4.2) ).

Remark 1.2. Since

$$
\left(\begin{array}{l}
Z(s, t ; u) \\
Z(t, u ; s) \\
Z(u, s ; t)
\end{array}\right)=\left(\begin{array}{ccc}
1 & \cos (\pi t) & \cos (\pi s) \\
\cos (\pi t) & 1 & \cos (\pi u) \\
\cos (\pi s) & \cos (\pi u) & 1
\end{array}\right)\left(\begin{array}{c}
\zeta(s, t ; u) \\
\zeta(t, u ; s) \\
\zeta(u, s ; t)
\end{array}\right)
$$

we can write $\zeta(s, t ; u)$ in terms of the $Z$-function as

$$
\begin{aligned}
\Delta(s, t, u) \zeta(s, t ; u)= & \left(1-\cos ^{2}(\pi u)\right) Z(s, t ; u) \\
& +(\cos (\pi s) \cos (\pi u)-\cos (\pi t)) Z(t, u ; s) \\
& +(\cos (\pi t) \cos (\pi u)-\cos (\pi s)) Z(u, s ; t),
\end{aligned}
$$


where $\Delta(s, t, u)=1-\cos ^{2}(\pi s)-\cos ^{2}(\pi t)-\cos ^{2}(\pi u)+2 \cos (\pi s) \cos (\pi t) \cos (\pi u)$, and so Theorem gives a new integral representation of $\zeta(s, t ; u)$. Some special cases will be displayed in Proposition 4.1 .

In this paper, to prove Theorem, we employ Li's method in [6] which gave a simple proof of (1.2). In $\S 2$, we will generalize some partial fraction decompositions used there to a usable form in our case. We will give a proof of Theorem in $\S 3$ and exhibit its applications in $\S 4$. In Appendix, we will show a functional equation for Euler's double zeta function.

\section{Generalized partial fRaCtion DeComposition}

The following partial fraction decomposition plays a fundamental role in the theory of multiple zeta values: for two independent variables $p, q$ and two positive integers $s, t$,

$$
\frac{1}{p^{s} q^{t}}=\sum_{h=0}^{s-1} \frac{(t)_{h}}{h !} \frac{1}{p^{s-h}(p+q)^{t+h}}+\sum_{k=0}^{t-1} \frac{(s)_{k}}{k !} \frac{1}{q^{t-k}(p+q)^{s+k}} .
$$

In this section, we will formulate two partial fraction decompositions in the case of $s, t$ being complex numbers.

Lemma 2.1. Let $p, q$ be positive real numbers and let $s, t$ be complex numbers whose real parts are positive. If $s, t \neq 1,2, \ldots$, then

$$
\frac{\Gamma(s) \Gamma(t)}{p^{s} q^{t}}=I(s, t ; p, r)+I(t, s ; q, r)
$$

where $r=p+q$ and

$$
I(s, t ; p, r)=\frac{1}{2 \pi i} \int_{L_{s, t}} \frac{\Gamma(1-s+\eta) \Gamma(-\eta)}{\Gamma(1-s)} \frac{\Gamma(s-\eta)}{p^{s-\eta}} \frac{\Gamma(t+\eta)}{r^{t+\eta}} d \eta .
$$

Here, the contour $L_{s, t}$ is a line from $-i \infty$ to $i \infty$ indented in such a manner as to separate the points at $\eta=s-1-m,-t-m(m=0,1,2, \ldots)$ from the points at $\eta=s+n, n(n=0,1,2, \ldots)$.

Proof. From the usual integral representation of the gamma function, it follows that

$$
I(s, t ; p, r)=\frac{1}{2 \pi i} \int_{L_{s, t}} \frac{\Gamma(1-s+\eta) \Gamma(-\eta)}{\Gamma(1-s)}\left(\iint_{\left.\mathbb{R}_{>0}\right)^{2}} e^{-p \mu-r \nu} \mu^{s-\eta-1} \nu^{t+\eta-1} d \mu d \nu\right) d \eta .
$$

By a suitable choice of $L_{s, t}$, it can be shown that the order of the integrations can be interchanged. Hence, we see

$$
I(s, t ; p, r)=\iint_{\left(\mathbb{R}_{>0}\right)^{2}} e^{-p \mu-r \nu} \mu^{s-1} \nu^{t-1}\left(\frac{1}{2 \pi i} \int_{L_{s, t}} \frac{\Gamma(1-s+\eta) \Gamma(-\eta)}{\Gamma(1-s)}(\nu / \mu)^{\eta} d \eta\right) d \mu d \nu .
$$

Since the innermost integral is $(1+\nu / \mu)^{s-1}$ (see [15, $\S 14.51$, Corollary]), we obtain

$$
\begin{aligned}
I(s, t ; p, r) & =\iint_{\left(\mathbb{R}_{>0}\right)^{2}} e^{-p \mu-r \nu}(\mu+\nu)^{s-1} \nu^{t-1} d \mu d \nu \\
& =\iint_{0<\nu<\mu} e^{-p \mu-q \nu} \mu^{s-1} \nu^{t-1} d \mu d \nu .
\end{aligned}
$$


We note that

$$
I(t, s ; q, r)=\iint_{0<\mu<\nu} e^{-p \mu-q \nu} \mu^{s-1} \nu^{t-1} d \mu d \nu,
$$

and so the right hand side of (2.2) is equal to

$$
\iint_{(\mathbb{R}>0)^{2}} e^{-p \mu-q \nu} \mu^{s-1} \nu^{t-1} d \mu d \nu=\frac{\Gamma(s) \Gamma(t)}{p^{s} q^{t}} .
$$

This is the desired result.

Lemma 2.2. Let $p, q, s, t$ be as in Lemma 2.1. If $p<q$ and $s, t \neq 1,2, \ldots$, then

$$
\frac{\cos (\pi s) \Gamma(s) \Gamma(t)}{p^{s} q^{t}}=J(s, t ; p, q-p)+I(t, s ; q, q-p),
$$

where

$$
J(s, t ; p, q-p)=\frac{1}{2 \pi i} \int_{L_{s, t}} \frac{\Gamma(1-s+\eta) \Gamma(-\eta)}{\Gamma(1-s)} \frac{\cos (\pi(s-\eta)) \Gamma(s-\eta)}{p^{s-\eta}} \frac{\Gamma(t+\eta)}{(q-p)^{t+\eta}} d \eta .
$$

Proof. For any $p, r \in \mathbb{C}^{\times}$, the integrand in (2.3) is

$$
\ll|\eta|^{\operatorname{Re}(t)-1} e^{-2 \pi|\eta|} e^{\operatorname{Im}(\eta)(\arg r-\arg p)}\left|p^{-s}\right|\left|r^{-t}\right|
$$

as $\eta \rightarrow \pm i \infty$ on $L_{s, t}$, where the implied constant does not depend on $p, r, \eta$. This estimate ensures that, for any fixed $q \in \mathbb{R}_{>0}$, the right hand side of (2.2) is continued to $\mathbb{C} \backslash\left\{ \pm i \mathbb{R}_{\geq 0} \cup\right.$ $\left.\left(-q \pm i \mathbb{R}_{\geq 0}\right)\right\}$ as a holomorphic function in $p$, where the double-signs correspond, and hence, if $0<p<q$, then

$$
\frac{e^{ \pm \pi i s} \Gamma(s) \Gamma(t)}{p^{s} q^{t}}=\frac{\Gamma(s) \Gamma(t)}{(-p)^{s} q^{t}}=I(s, t ;-p, q-p)+I(t, s ; q, q-p)
$$

and

$$
I(s, t ;-p, q-p)=\frac{1}{2 \pi i} \int_{L_{s, t}} \frac{\Gamma(1-s+\eta) \Gamma(-\eta)}{\Gamma(1-s)} \frac{e^{ \pm \pi i(s-\eta)} \Gamma(s-\eta)}{p^{s-\eta}} \frac{\Gamma(t+\eta)}{(q-p)^{t+\eta}} d \eta .
$$

Thus, we obtain Lemma 2.2 .

\section{Proof of Theorem}

For simplicity of description, we suppose that $\operatorname{Re}(s), \operatorname{Re}(t), \operatorname{Re}(u)>2, s, t \neq 3,4,5, \ldots$ and the contour $L_{s, t}$ always satisfies the condition $-1 / 2 \leq \operatorname{Re}(\eta) \leq \operatorname{Re}(s)-1 / 2$ for all $\eta \in L_{s, t}$. We first evaluate

$$
\zeta(s, t ; u)=\sum_{m, n=1}^{\infty} \frac{1}{m^{s} n^{t}(m+n)^{u}} .
$$

Applying Lemma 2.1 with $(p, q)=(m, n)$, we see

$$
\zeta(s, t ; u)=X(s, t ; u)+X(t, s ; u),
$$

where

$$
X(s, t ; u)=\frac{1}{2 \pi i} \sum_{m, n=1}^{\infty} \int_{L_{s, t}} \frac{\Gamma(s, t ; \eta)}{m^{s-\eta}(m+n)^{t+u+\eta}} d \eta
$$


and

$$
\Gamma(s, t ; \eta)=\frac{\Gamma(1-s+\eta) \Gamma(-\eta) \Gamma(s-\eta) \Gamma(t+\eta)}{\Gamma(1-s) \Gamma(s) \Gamma(t)} .
$$

From the condition of $L_{s, t}$, it follows that the order of summation and integration of $X(s, t ; u)$ can be interchanged. As a result, we have

$$
\begin{aligned}
X(s, t ; u)= & \frac{1}{2 \pi i} \int_{L_{s, t}} \Gamma(s, t ; \eta) \zeta(s-\eta, 0 ; t+u+\eta) d \eta \\
= & \frac{\Gamma(s+t-1)}{\Gamma(s) \Gamma(t)} \zeta(1,0 ; s+t+u-1) \\
& +\frac{1}{2 \pi i} \int_{L_{s-1, t}} \Gamma(s, t ; \eta) \zeta(s-\eta, 0 ; t+u+\eta) d \eta .
\end{aligned}
$$

We next treat $\cos (\pi t) \zeta(t, u ; s)+\cos (\pi s) \zeta(u, s ; t)$. Set

$$
a_{m, n}(s, t ; u)=\frac{\cos (\pi t)}{m^{t} n^{u}(m+n)^{s}}+\frac{\cos (\pi s)}{n^{u} m^{s}(m+n)^{t}}
$$

for $m, n \in \mathbb{Z}_{\geq 1}$. Applying Lemma 2.2 to each term, we obtain

$$
a_{m, n}(s, t ; u)=b_{m, n}(s, t ; u)+b_{m, n}(t, s ; u),
$$

where

$$
\begin{aligned}
b_{m, n}(s, t ; u)= & \frac{I(s, t ; m+n, n)+J(s, t ; m, n)}{\Gamma(s) \Gamma(t) n^{u}} \\
= & -\frac{\Gamma(s+t-1)}{\Gamma(s) \Gamma(t)} \frac{1}{n^{s+t+u-1}}\left(\frac{1}{m}-\frac{1}{m+n}\right) \\
& +\frac{1}{2 \pi i} \int_{L_{s-1, t}} \Gamma(s, t ; \eta)\left(\frac{1}{n^{t+u+\eta}(m+n)^{s-\eta}}+\frac{\cos (\pi(s-\eta))}{m^{s-\eta} n^{t+u+\eta}}\right) d \eta .
\end{aligned}
$$

Put $Y(s, t ; u)=\sum_{m, n=1}^{\infty} b_{m, n}(s, t ; u)$. Then, it is easily seen that

$$
\cos (\pi t) \zeta(t, u ; s)+\cos (\pi s) \zeta(u, s ; t)=Y(s, t ; u)+Y(t, s ; u)
$$

and that

$$
\begin{aligned}
Y(s, t ; u)= & -\frac{\Gamma(s+t-1)}{\Gamma(s) \Gamma(t)}(\zeta(1,0 ; s+t+u-1)+\zeta(s+t+u)) \\
& +\frac{1}{2 \pi i} \int_{L_{s-1, t}} \Gamma(s, t ; \eta)\{\zeta(t+u+\eta, 0 ; s-\eta) \\
& +\cos (\pi(s-\eta)) \zeta(s-\eta) \zeta(t+u+\eta)\} d \eta
\end{aligned}
$$


Combining (3.1) and (3.2), we have

$$
\begin{aligned}
X(s, t ; u)+Y(s, t ; u) & \\
= & -\frac{\Gamma(s+t-1)}{\Gamma(s) \Gamma(t)} \zeta(s+t+u) \\
& +\frac{1}{2 \pi i} \int_{L_{s-1, t}} \Gamma(s, t ; \eta)\{1+\cos (\pi(s-\eta))\} \zeta(s-\eta) \zeta(t+u+\eta) d \eta \\
& -\frac{1}{2 \pi i} \int_{L_{s-1, t}} \Gamma(s, t ; \eta) d \eta \zeta(s+t+u)
\end{aligned}
$$

because generally

$$
\zeta(s, 0 ; t)+\zeta(t, 0 ; s)=\zeta(s) \zeta(t)-\zeta(s+t)
$$

The second term on the right hand side of $(\underline{3.3})$ becomes

$$
\frac{\Gamma(s+t)}{s \Gamma(s) \Gamma(t)} \zeta(s+t+u)+A(s, t ; u)
$$

by shifting the contour to $L_{s+1, t}$, and the third term is

$$
\begin{aligned}
& =\frac{\Gamma(s+t-1)}{\Gamma(s) \Gamma(t)} \zeta(s+t+u)-\frac{1}{2 \pi i} \int_{L_{s, t}} \Gamma(s, t ; \eta) d \eta \zeta(s+t+u) \\
& =\frac{\Gamma(s+t-1)}{\Gamma(s) \Gamma(t)} \zeta(s+t+u)-\frac{\Gamma(s+t)}{t \Gamma(s) \Gamma(t)} \zeta(s+t+u)
\end{aligned}
$$

by Barnes' lemma (see [15, 14.52]). Hence,

$$
X(s, t ; u)+Y(s, t ; u)=\left(\frac{1}{s}-\frac{1}{t}\right) \frac{\Gamma(s+t)}{\Gamma(s) \Gamma(t)} \zeta(s+t+u)+A(s, t ; u) .
$$

Thus, we have

$$
\begin{aligned}
Z(s, t ; u) & =X(s, t ; u)+X(t, s ; u)+Y(s, t ; u)+Y(t, s ; u) \\
& =A(s, t ; u)+A(t, s ; u),
\end{aligned}
$$

when $\operatorname{Re}(s), \operatorname{Re}(t), \operatorname{Re}(u)>2$ and $s, t \neq 3,4,5, \ldots$ By the theory of analytic continuation, the proof of Theorem is completed.

Remark 3.1. We have used Li's method in this section, but it is possible to prove Theorem by Nakamura's original method. Indeed, all his argument is valid here except for the property for the Bernoulli polynomial [9, $(2,7)]$, which can be proved by the partial fractional decomposition (2.1) in a similar way to Eisenstein's proof for addition formulas of the trigonometric functions (see [14, Chapter II] or [11, §2.1]). And so it can be reformulated to an available form in our case by (a slightly generalized) Lemma 2.1.

\section{Application}

In this section, we will find some new results from Theorem. The following each proposition can be proved independently of the others. However, the next lemma seems to be useful for some applications, and so we first state it in order to access quickly. 
Lemma 4.1. Let $a$ be an integer and $b, c$ be non-negative integers. Set

$$
F(s, t ; c)=\sum_{k=0}^{c}\left(\begin{array}{l}
c \\
k
\end{array}\right) \zeta(s-k) \zeta(t-c+k)
$$

for $s, t \in \mathbb{C}$. Then we have the followings:

(1) For $t, u \in \mathbb{C}$ with $t+u \neq 1-l(l=0,1,2, \ldots)$,

$$
A(a, t ; u)=2 \sum_{k=0}^{[a / 2]}\left(\begin{array}{c}
t+a-2 k-1 \\
a-2 k
\end{array}\right) \zeta(2 k) \zeta(t+u+a-2 k),
$$

where the value of any empty sum is defined to be 0 .

(2) For $s, u \in \mathbb{C}$ with $u \neq b+1-l(l=0,1,2, \ldots)$,

$$
A(s,-b ; u)=\sum_{k=0}^{b}\left(\begin{array}{l}
b \\
k
\end{array}\right)\left(\cos (\pi s)+(-1)^{k}\right) \zeta(s-k) \zeta(u-b+k) .
$$

(3) For any $s \in \mathbb{C}$,

$$
\lim _{u \rightarrow-c} A(s,-b ; u)=\left(\cos (\pi s)-(-1)^{b+c}\right) F(s,-c ; b)+\delta_{c 0}(-1)^{b+1} \zeta(s-b),
$$

where $\delta_{i j}$ denotes the Kronecker symbol.

(4) For any $s \in \mathbb{C}$,

$$
\begin{aligned}
& \lim _{t \rightarrow-b} A(s, t ;-c) \\
&=\left(\cos (\pi s)-(-1)^{b+c}\right)\left(F(s,-c ; b)+\frac{(-1)^{c+1} b ! c !}{(b+c+1) !} \zeta(s-b-c-1)\right) \\
& \quad+\delta_{c 0}(-1)^{b+1} \zeta(s-b) .
\end{aligned}
$$

Proof. (1),(2) The formulas follow immediately from (1.4).

(3) We apply (2) to get

$$
\begin{aligned}
\lim _{u \rightarrow-c} A(s,-b ; u)= & \left(\cos (\pi s)-(-1)^{b+c}\right) \sum_{k=0}^{b-1}\left(\begin{array}{l}
b \\
k
\end{array}\right) \zeta(s-k) \zeta(-b-c+k) \\
& +\left(\cos (\pi s)+(-1)^{b}\right) \zeta(s-b) \zeta(-c),
\end{aligned}
$$

where we have used the fact that $\zeta(-b-c+k)=0$ if $0 \leq k \leq b-1$ and $k \equiv b+c(\bmod 2)$. Thus, by simple calculation, we obtain the result.

(4) The result follows in a similar way to the above.

We give integral representations of several zeta functions.

Proposition 4.1. (1) The Euler double zeta function $\zeta(s, t)$ has the following representation:

$$
\begin{aligned}
& (\cos (\pi t)-\cos (\pi s)) \zeta(s, t) \\
& \quad=A(s, t ; 0)+A(t, s ; 0)-(1+\cos (\pi s)) \zeta(s) \zeta(t)+\cos (\pi s) \zeta(s+t) .
\end{aligned}
$$


(2) Let $n$ be a non-negative integer. Then,

$$
\begin{aligned}
(1 & +\cos (\pi s)) \zeta(s) \zeta(s+2 n) \\
& =A(s, s+2 n ; 0)+A(s+2 n, s ; 0)+\cos (\pi s) \zeta(2 s+2 n)
\end{aligned}
$$

and

$$
\begin{aligned}
& (1+\cos (\pi s)) \zeta(s) \zeta(-s+2 n) \\
& \quad=A(s,-s+2 n ; 0)+A(-s+2 n, s ; 0)+\cos (\pi s) \zeta(2 n)+\delta_{n}(s)
\end{aligned}
$$

where

In particular, we obtain

$$
\delta_{n}(s)= \begin{cases}-\pi s \sin (\pi s) / 12 & \text { if } n=0 \\ -\pi \sin (\pi s) /(s-1) & \text { if } n=1 \\ 0 & \text { otherwise }\end{cases}
$$

$$
\begin{aligned}
(1 & +\cos (\pi s)) \zeta(s)^{2}-\cos (\pi s) \zeta(2 s)=2 A(s, s ; 0) \\
& =\frac{2 \sin (\pi s)}{2 \pi i} \int_{L} \cot \left(\frac{\pi(s-\eta)}{2}\right) \frac{\Gamma(s+\eta) \Gamma(-\eta)}{\Gamma(s)} \zeta(s-\eta) \zeta(s+\eta) d \eta
\end{aligned}
$$

(3) The Witten zeta function of $S U(3)$ can be written as

$$
\begin{aligned}
& 2^{-s-1}(1+2 \cos (\pi s)) \zeta_{S U(3)}(s)=A(s, s ; s) \\
& \quad=\frac{\sin (\pi s)}{2 \pi i} \int_{L} \cot \left(\frac{\pi(s-\eta)}{2}\right) \frac{\Gamma(s+\eta) \Gamma(-\eta)}{\Gamma(s)} \zeta(s-\eta) \zeta(2 s+\eta) d \eta .
\end{aligned}
$$

Remark 4.1. We can regard (4.4) as a generalization of the formula

$$
\begin{aligned}
& \zeta(2 l) \zeta(2 m)-\frac{1}{2} \zeta(2 l+2 m) \\
& \quad=\sum_{k=0}^{\max \{l, m\}}\left\{\left(\begin{array}{c}
2 l+2 m-2 k-1 \\
2 l-1
\end{array}\right)+\left(\begin{array}{c}
2 l+2 m-2 k-1 \\
2 m-1
\end{array}\right)\right\} \zeta(2 k) \zeta(2 l+2 m-2 k)
\end{aligned}
$$

for $l, m \in \mathbb{Z}_{\geq 1}$. Indeed, taking $s=2 l(l=1,2, \ldots)$ in (4.4) and putting $m=l+n$, this follows from (4.2).

Proof of Proposition 4.1. (1) Substituting $u=0$ in (1.1) and using (3.4), we see

$$
\begin{aligned}
Z(s, t ; 0) & =\zeta(s) \zeta(t)+\cos (\pi t) \zeta(t, 0 ; s)+\cos (\pi s) \zeta(0, s ; t) \\
& =(\cos (\pi t)-\cos (\pi s)) \zeta(t, 0 ; s)+(1+\cos (\pi s)) \zeta(s) \zeta(t)-\cos (\pi s) \zeta(s+t) .
\end{aligned}
$$

Hence, the result is shown by Theorem.

(2) Assume that $\operatorname{Re}(s)>1$. Comparing the limits of the both sides of (4.3) as $t \rightarrow \pm s+2 n$, we get

$$
\begin{aligned}
(1+ & \cos (\pi s)) \zeta(s) \zeta( \pm s+2 n) \\
= & A(s, \pm s+2 n ; 0)+A( \pm s+2 n, s ; 0)+\cos (\pi s) \zeta(s \pm s+2 n) \\
& -\lim _{z \rightarrow 0}\{\cos (\pi(z \pm s))-\cos (\pi s)\} \zeta(z \pm s+2 n, 0 ; s)
\end{aligned}
$$


where the double-signs correspond. It is clear that the limit becomes 0 unless the double-signs are "-" and $n=0,1$. In the remaining cases, the last term of the right side is $\delta_{n}(s)$ because

$$
\zeta(z-s+2 n, 0 ; s)=\frac{1}{s-1} \zeta(z+2 n-1)+\frac{s}{12} \zeta(z+2 n+1)+O(1)
$$

as $z \rightarrow 0$ (see [7, p.425, (4.4)]).

(3) The result follows immediately from (1.1) and Theorem.

We next extend the parity result [2, Theorem 2] to the whole domain of convergence.

Proposition 4.2. Let $a, b, c$ be integers such that $a+b+c$ is odd. Assume that $a+c \geq 2$, $b+c \geq 2$ and $a+b+c \geq 3$. If $a+b \geq 2$, then

$$
2 \zeta(a, b ; c)=(-1)^{a}\{A(c, a ; b)+A(a, c ; b)\}+(-1)^{b}\{A(c, b ; a)+A(b, c ; a)\},
$$

where every $A$-value is representable in the form (4.2). If $a+b \leq 1$, then

$$
\begin{aligned}
2 \zeta(a, b ; c)= & (-1)^{a}\left\{A^{*}(c, a ; b)+A(a, c ; b)\right\}+(-1)^{b}\left\{A^{*}(c, b ; a)+A(b, c ; a)\right\} \\
& +\left.\frac{(-1)^{a} 2}{(1-a-b) !} \frac{d}{d s}(s+a)_{1-a-b}\right|_{s=0} \zeta(a+b+c-1) .
\end{aligned}
$$

Here

$$
A^{*}(c, a ; b)=2 \sum_{k}\left(\begin{array}{c}
a+c-2 k-1 \\
c-2 k
\end{array}\right) \zeta(2 k) \zeta(a+b+c-2 k),
$$

where the sum is taken over all integers $k \in[0, c / 2]$ except for $k=(a+b+c-1) / 2$.

Proof. Taking the limits of the both sides of (1.5) as $u \rightarrow c, t \rightarrow b$ and $s \rightarrow a$ in order, we have

$$
\begin{aligned}
2 \zeta(a, b ; c)= & (-1)^{a} A(a, c ; b)+(-1)^{b} A(b, c ; a) \\
& +\lim _{s \rightarrow a}\left((-1)^{a} A(c, s ; b)+(-1)^{b} A(c, b ; s)\right) .
\end{aligned}
$$

It is easily seen that the limiting value equals $(-1)^{a} A(c, a ; b)+(-1)^{b} A(c, b ; a)$ if $a+b \geq 2$, and

$$
\begin{aligned}
& (-1)^{a} A^{*}(c, a ; b)+(-1)^{b} A^{*}(c, b ; a) \\
& +\left.\frac{(-1)^{a} 2}{(1-a-b) !} \frac{d}{d s}(s+a)_{1-a-b}\right|_{s=0} \zeta(a+b+c-1)
\end{aligned}
$$

if $a+b \leq 1$. Thus, we obtain Proposition 4.2

The following proposition suggests that $\zeta(s, t ; u)$ can be represented as a sum of products of the Riemann zeta functions, if at least two of $s, t$ and $u$ are non-positive integers in the sense of the coordinate-wise limit.

Proposition 4.3. Let $a, b$ and $c$ be non-negative integers and let $s, t$ and $u$ be complex numbers.

(1) If $s, t \neq c+1-l(l=0,1,2, \ldots)$ and $s+t \neq c+2$, then $\zeta(s, t ;-c)=F(s, t ; c)$, where $F(s, t ; c)$ is defined by (4.1). 
(2) If $u \neq a+1-l, b+1-l, a+b+2(l=0,1,2, \ldots)$, then

$$
\begin{aligned}
\zeta(-a,-b ; u)= & (-1)^{a+1} F(u,-a ; b)+(-1)^{b+1} F(u,-b ; a) \\
& +\frac{a ! b !}{(a+b+1) !} \zeta(u-a-b-1)-\delta_{a 0} \zeta(u-b)-\delta_{b 0} \zeta(u-a) .
\end{aligned}
$$

(3) For $s \in \mathbb{C}$ with $s \neq c+1-l, b+c+2(l=0,1,2, \ldots)$,

$$
\lim _{u \rightarrow-c} \zeta(s,-b ; u)=F(s,-b ; c)+\frac{(-1)^{b+1} b ! c !}{(b+c+1) !} \zeta(s-b-c-1) .
$$

Proof. (1) This is trivial.

(2) We take the limits of the both sides of (1.5) as $t \rightarrow-b$ and $s \rightarrow-a$ in order. Then, the result is a direct consequence of Lemma 4.1 .

(3) The result can be proved in a similar way to (2), but it follows easily from the representation [7, (5.3)] of $\zeta(s, t ; u)$.

Corollary 4.1. Let $a, b$ and $c$ be non-negative integers.

$$
\lim _{(s, t) \rightarrow(-a,-b)} \zeta(s, t ;-c)=F(-a,-b ; c) .
$$

$$
\lim _{u \rightarrow-c} \zeta(-a,-b ; u)=F(-a,-b ; c)+\left(\frac{(-1)^{a+1} a ! c !}{(a+c+1) !}+\frac{(-1)^{b+1} b ! c !}{(b+c+1) !}\right) \zeta(-a-b-c-1) .
$$

$$
\begin{aligned}
& \lim _{s \rightarrow-a} \lim _{u \rightarrow-c} \zeta(s,-b ; u)=F(-a,-b ; c)+\frac{(-1)^{b+1} b ! c !}{(b+c+1) !} \zeta(-a-b-c-1) . \\
& \lim _{t \rightarrow-b} \lim _{u \rightarrow-c} \zeta(-a, t ; u)=F(-a,-b ; c)+\frac{(-1)^{a+1} a ! c !}{(a+c+1) !} \zeta(-a-b-c-1) .
\end{aligned}
$$

Remark 4.2. Komori [3] studied Tornheim's double zeta values for coordinate-wise limits at nonpositive integers and gave their explicit expressions in terms of generalized Bernoulli numbers. Our formulation seems to be more concrete than his.

To prove (2), we have to use the following lemma and the relation

$$
(-1)^{a+b+c} F(-a,-b ; c)-\delta_{a 0} \zeta(-b-c)-\delta_{b 0} \zeta(-a-c)-\delta_{a 0} \delta_{b 0} \delta_{c 0}=F(-a,-b ; c) .
$$

Lemma 4.2. Let $a, b$ and $c$ be non-negative integers. Then,

$$
\begin{aligned}
& (-1)^{a+b} F(-a,-b ; c)+(-1)^{b+c} F(-b,-c ; a)+(-1)^{c+a} F(-c,-a ; b) \\
& =\left(\frac{(-1)^{c} a ! b !}{(a+b+1) !}+\frac{(-1)^{a} b ! c !}{(b+c+1) !}+\frac{(-1)^{b} c ! a !}{(c+a+1) !}\right) \zeta(-a-b-c-1) \\
& \quad+\delta_{a 0} \delta_{b 0} \delta_{c 0} .
\end{aligned}
$$

This lemma is equivalent to Theorem 2 of $\mathrm{Chu}$-Wang [1. However, their formulation is quite different from ours, and so we now prove it for the reader's convenience. 
Proof. For a non-negative integer $m$, we set

$$
\tilde{P}_{m}(x)=\delta_{m 0}+(-1)^{m} \frac{m !}{x^{m+1}}+2^{m+1} \sum_{k=0}^{\infty}(-1)^{m+k} \zeta(-m-k) \frac{(2 x)^{k}}{k !} .
$$

We calculate the value

$$
R=2^{-a-b-c-2}\left[x^{-1}\right]\left(\tilde{P}_{a}(x)-\delta_{a 0}\right)\left(\tilde{P}_{b}(x)-\delta_{b 0}\right)\left(\tilde{P}_{c}(x)-\delta_{c 0}\right)
$$

in two ways, where $\left[x^{-1}\right] f(x)$ denotes the formal residue of a formal Laurent series $f(x)$. We first use the definition of $\tilde{P}_{m}(x)$ to obtain

$$
\begin{aligned}
R= & (-1)^{a+b} F(-a,-b ; c)+(-1)^{b+c} F(-b,-c ; a)+(-1)^{c+a} F(-c,-a ; b) \\
& -\left(\frac{(-1)^{c} a ! b !}{(a+b+1) !}+\frac{(-1)^{a} b ! c !}{(b+c+1) !}+\frac{(-1)^{b} c ! a !}{(c+a+1) !}\right) \zeta(-a-b-c-1) .
\end{aligned}
$$

We next apply Proposition 3.1 in [10] to get $R=\delta_{a 0} \delta_{b 0} \delta_{c 0}$. Thus, we have (4.6).

We finally show the behavior of $\zeta_{S U(3)}(s)$ at each integer.

Proposition 4.4. Let a be a positive integer.

(1) [2, Theorem 3]

$$
\zeta_{S U(3)}(a)=\frac{2^{a+2}}{1+(-1)^{a} 2} \sum_{k=0}^{[a / 2]}\left(\begin{array}{c}
2 a-2 k-1 \\
a-1
\end{array}\right) \zeta(2 k) \zeta(3 a-k) .
$$

(2) $\zeta_{S U(3)}(0)=1 / 3$ and $\zeta_{S U(3)}^{\prime}(0)=\log \left(2^{4 / 3} \pi\right)$.

(3) If a is odd, then $\zeta_{S U(3)}(s)$ has a simple zero at $s=-a$, and

$$
\zeta_{S U(3)}^{\prime}(-a)=2^{-a+2} \sum_{k=0}^{(a-1) / 2}\left(\begin{array}{c}
a \\
2 k
\end{array}\right) \zeta(-a-2 k) \zeta^{\prime}(-2 a+2 k)+\frac{2^{-a+1}(a !)^{2}}{(2 a+1) !} \zeta^{\prime}(-3 a-1) .
$$

In particular, $\operatorname{sign}\left(\zeta_{S U(3)}^{\prime}(-a)\right)=(-1)^{(a-1) / 2}$.

(4) If a is even, then $\zeta_{S U(3)}(s)$ has a zero of order two at $s=-a$, and

$$
\zeta_{S U(3)}^{\prime \prime}(-a)=2^{-a+2} \sum_{k=0}^{a / 2}\left(\begin{array}{c}
a \\
2 k
\end{array}\right) \zeta^{\prime}(-a-2 k) \zeta^{\prime}(-2 a+2 k) .
$$

In particular, $\operatorname{sign}\left(\zeta_{S U(3)}^{\prime \prime}(-a)\right)=(-1)^{a / 2}$.

Remark 4.3. The value of Witten's zeta function $\zeta_{G}(s)$ of each finite group $G$ at $s=-2$ coincides with the order of $G$. In this viewpoint, it is attractive to clarify the behavior of $\zeta_{G}(s)$ at $s=-2$ in the case of $G$ being an infinite compact topological group. In [5], Kurokawa and Ochiai studied the values of Witten's zeta functions at negative integers, and proved that $\zeta_{S U(3)}(s)$ has a zero at each negative integer. Proposition 4.4 can be regarded as a refinement of their result. Moreover, as seen below, our proof reveals that a zero of $\zeta_{S U(3)}(s)$ at each negative integer comes from the gamma factors appearing in the left sides of (2.2) and (2.4). 
Proof of Proposition 4.4. We use here the integral representation (4.5) of $\zeta_{S U(3)}(s)$.

(1) The result is clear from (4.2).

(2) By (1.4), we see that, if $K$ is a non-negative integer and $-K / 2+1 / 4<\operatorname{Re}(s)<K+1 / 2$, then

$$
\begin{aligned}
& 2^{-s-1}(1+2 \cos (\pi s)) \zeta_{S U(3)}(s) \\
& \quad=2 \sum_{k=0}^{K} \frac{(s)_{k}}{k !} \cos ^{2}\left(\frac{\pi(s-k)}{2}\right) \zeta(s-k) \zeta(2 s+k)+\frac{\sin (\pi s)}{\Gamma(s)} R_{K}(s),
\end{aligned}
$$

where $R_{K}(s)$ is a holomorphic function. We note that every term except for the term with $k=0$ has a zero of order at least two at $s=0$. Hence, the values at $s=0$ can be immediately calculated.

(3) Set $K=2 a+1$. If $a$ is odd, then the terms with $k=0,1, \ldots, a$ satisfying $k \equiv a(\bmod 2)$ and the term with $k=2 a+1$ have a simple zero at $s=-a$, and the others have a zero of order at least two. Hence, we can easily obtain the first part of the result. The last part follows from the functional equation of the Riemann zeta function. Indeed, we can show that the sign of each term on the right side of (4.7) coincides with $(-1)^{(a-1) / 2}$.

(4) Put $K=2 a+1$ again. In the same way, we see that $\zeta_{S U(3)}(s)$ has a zero of order at least two at $s=-a$ if $a$ is even. In order to determine the multiplicity of the zero, we now show (4.8). Assume that $0<\varepsilon<1 / 2$. Set

$$
f(s, \eta)=\frac{\sin (\pi s)}{\Gamma(s)} \cot \left(\frac{\pi(s-\eta)}{2}\right) \Gamma(s+\eta) \Gamma(-\eta) \zeta(s-\eta) \zeta(2 s+\eta) .
$$

Then, by shifting the contour, we obtain the following expression of $\zeta_{S U(3)}(s)$ which is valid around $s=-a$ :

$$
2^{-s-1}(1+2 \cos (\pi s)) \zeta_{S U(3)}(s)=-\sum_{k=0}^{a / 2} U_{k}(s)+\sum_{l=0}^{a / 2-1} V_{l}(s)+W(s)+I(s),
$$

where $U_{k}(s)=\operatorname{Res}_{\eta=k} f(s, \eta), V_{l}(s)=\operatorname{Res}_{\eta=-s-l} f(s, \eta), W(s)=\operatorname{Res}_{\eta=1-2 s} f(s, \eta)$ and

$$
I(s)=\frac{1}{2 \pi i} \int_{C_{\varepsilon}} f(s, \eta) d \eta
$$

whose contour $C_{\varepsilon}$ describes the union of $C_{\varepsilon}^{(1)}: a / 2-i \infty \rightarrow a / 2-i \varepsilon, C_{\varepsilon}^{(2)}: a / 2+\varepsilon e^{i \theta}(\theta$ : $-\pi / 2 \rightarrow \pi / 2)$ and $C_{\varepsilon}^{(3)}: a / 2+i \varepsilon \rightarrow a / 2+i \infty$. We here remark that the poles at $\eta=$ $k, s-2 m,-s-a / 2-m(k=0,1, \ldots, a / 2 ; m=0,1,2, \ldots)$ lie on the left of the contour $C_{\varepsilon}$ and the poles at $\eta=1-2 s,-s-l, a / 2+n(l=0,1, \ldots, a / 2-1 ; n=1,2, \ldots)$ lie on the right. Hence,

$$
2^{a-1} 3 \zeta_{S U(3)}^{\prime \prime}(-a)=-\sum_{k=0}^{a / 2} U_{k}^{\prime \prime}(-a)+\sum_{l=0}^{a / 2-1} V_{l}^{\prime \prime}(-a)+W^{\prime \prime}(-a)+I^{\prime \prime}(-a) .
$$


By simple calculation, we first see that, for $k=0,1, \ldots, a / 2$ and $l=0,1, \ldots, a / 2-1$,

$$
\begin{aligned}
& U_{k}^{\prime \prime}(-a)= \begin{cases}-8\left(\begin{array}{l}
a \\
k
\end{array}\right) \zeta^{\prime}(-a-k) \zeta^{\prime}(-2 a+k) & \text { if } k \text { is even, } \\
\pi^{2}\left(\begin{array}{l}
a \\
k
\end{array}\right) \zeta(-a-k) \zeta(-2 a+k) & \text { if } k \text { is odd },\end{cases} \\
& V_{l}^{\prime \prime}(-a)= \begin{cases}4\left(\begin{array}{l}
a \\
l
\end{array}\right) \zeta^{\prime}(-a-l) \zeta^{\prime}(-2 a+l) & \text { if } l \text { is even, } \\
-2 \pi^{2}\left(\begin{array}{l}
a \\
l
\end{array}\right) \zeta(-a-l) \zeta(-2 a+l) & \text { if } l \text { is odd },\end{cases}
\end{aligned}
$$

and

$$
W^{\prime \prime}(-a)=\frac{3 \pi^{2}}{2} \frac{(a !)^{2}}{(2 a+1) !} \zeta(-3 a-1)
$$

We next evaluate

$$
I^{\prime \prime}(-a)=\frac{1}{2 \pi i} \int_{C_{\varepsilon}} f^{\prime \prime}(-a, \eta) d \eta
$$

where $f^{\prime \prime}$ means $(\partial / \partial s)^{2} f$. Since the integrals on $C_{\varepsilon}^{(1)}$ and $C_{\varepsilon}^{(3)}$ cancel each other out, we obtain

$$
\begin{aligned}
I^{\prime \prime}(-a)= & \frac{1}{2 \pi i} \int_{C_{\varepsilon}^{(2)}} \operatorname{Res}_{\eta=a / 2} f^{\prime \prime}(-a, \eta) \frac{d \eta}{\eta-a / 2} \\
& +\frac{1}{2 \pi i} \int_{C_{\varepsilon}^{(2)}}\left(f^{\prime \prime}(-a, \eta)-\operatorname{Res}_{\eta=a / 2} f^{\prime \prime}(-a, \eta) \cdot \frac{1}{\eta-a / 2}\right) d \eta .
\end{aligned}
$$

We note that the integrand in the second integral is holomorphic at $\eta=a / 2$, and so the second integral tends to zero as $\varepsilon$ tends to zero. Since $I(s)$ is independent of the choice of $\varepsilon$, we get

$$
I^{\prime \prime}(-a)=\frac{1}{2} \operatorname{Res}_{\eta=a / 2} f^{\prime \prime}(-a, \eta)=D_{1}(a)+D_{2}(a),
$$

where

$$
\begin{aligned}
& D_{1}(a)=\left\{\begin{array}{lll}
-\frac{\pi^{2}}{2}\left(\begin{array}{c}
a \\
a / 2
\end{array}\right) \zeta(-3 a / 2)^{2} & \text { if } a \equiv 2 & (\bmod 4), \\
0 & \text { if } a \equiv 0 & (\bmod 4),
\end{array}\right. \\
& D_{2}(a)=\left\{\begin{array}{lll}
0 & \text { if } a \equiv 2 & (\bmod 4), \\
-2\left(\begin{array}{c}
a \\
a / 2
\end{array}\right) \zeta^{\prime}(-3 a / 2)^{2} & \text { if } a \equiv 0 & (\bmod 4) .
\end{array}\right.
\end{aligned}
$$

Combining the above results, we have

$$
\begin{aligned}
& -\sum_{\substack{0 \leq k \leq a / 2 \\
k: \text { odd }}} U_{k}^{\prime \prime}(-a)+\sum_{\substack{0 \leq l \leq a / 2-1 \\
l: \text { odd }}} V_{l}^{\prime \prime}(-a)+W^{\prime \prime}(-a)+D_{1}(a) \\
& \quad=-\frac{3 \pi^{2}}{2} \sum_{k=0}^{a}\left(\begin{array}{l}
a \\
k
\end{array}\right) \zeta(-a-k) \zeta(-2 a+k)+\frac{3 \pi^{2}}{2} \frac{(a !)^{2}}{(2 a+1) !} \zeta(-3 a-1) \\
& \quad=0,
\end{aligned}
$$


where in the last step we have used (4.6) with $a=b=c$. Moreover, we see

$$
\begin{gathered}
-\sum_{\substack{0 \leq k \leq a / 2 \\
k: \text { even }}} U_{k}^{\prime \prime}(-a)+\sum_{\substack{0 \leq l \leq a / 2-1 \\
l: \text { even }}} V_{l}^{\prime \prime}(-a)+D_{2}(a) \\
=6 \sum_{k=0}^{a / 2}\left(\begin{array}{c}
a \\
2 k
\end{array}\right) \zeta^{\prime}(-a-2 k) \zeta^{\prime}(-2 a+2 k) .
\end{gathered}
$$

Thus, we obtain (4.8). In the same way as (3) above, we get $\operatorname{sign}\left(\zeta_{S U(3)}^{\prime \prime}(-a)\right)=(-1)^{a / 2}$, which completes the proof of Proposition 4.4.

\section{Appendix A. A functional equation for Euler's double zeta function}

The $A$-function (1.3) has not been found in previous papers on multiple zeta functions. However, as seen in the next proposition, $A(s, t ; 0)$ is related to the functional equation of $\zeta(s, t)=\zeta(t, 0 ; s)$ which was obtained by Matsumoto [8, Theorem 1].

Proposition A.1. Set

$$
h(s, t)=\zeta(s, t)-\frac{\Gamma(1-t)}{\Gamma(s)} \Gamma(s+t-1) \zeta(s+t-1) .
$$

Then, we have

$$
\begin{aligned}
\frac{h(s, t)}{(2 \pi)^{s+t-1} \Gamma(1-t)}= & \cos \left(\frac{\pi}{2}(s+t-1)\right) \frac{h(1-t, 1-s)}{\Gamma(s)} \\
& +\sin \left(\frac{\pi}{2}(s+t-1)\right) \frac{\Gamma(1-s)}{\pi} A(1-s, 1-t ; 0) .
\end{aligned}
$$

In particular, the second term on the right side of (A.1) vanishes on the hyperplane $s+t=2 k+1$ $(k \in \mathbb{Z} \backslash\{0\})$ (cf. [4, Theorem 2.2]).

Remark A.1. Firstly, the function $g(u, v)$ in Matsumoto's paper coincides with $h(v, u)$. Secondly, it may seem that the singularities of $\zeta(s, t)$ are located on the hyperplanes $s=1-l$ and $s+t=2-l(l=0,1,2, \ldots)$. However, the singularities on $s=-l$ and $s+t=-1-2 l(l=$ $0,1,2, \ldots)$ are fake, namely, the singularities of $\zeta(s, t)$ are only located on the hyperplanes $s=1, s+t=1$ and $s+t=2-2 l(l=0,1,2, \ldots)$. This can be confirmed, for instance, by (1.4) and (4.3). Hence, the last part of the proposition is justified.

Proof of Proposition A.1. We first recall the usual integral representation of $\zeta(s, t)$ (cf. [7, $(5.2)])$ :

$$
\zeta(s, t)=\frac{1}{2 \pi i} \int_{(c)} \frac{\Gamma(s+\eta) \Gamma(-\eta)}{\Gamma(s)} \zeta(t-\eta) \zeta(s+\eta) d \eta
$$

for $s, t \in \mathbb{C}$ with $\operatorname{Re}(s)>1$ and $\operatorname{Re}(t)>1$, where $-\operatorname{Re}(s)+1<c<0$ and the contour $(c)$ describes the line from $c-i \infty$ to $c+i \infty$. Since the residue of the integrand at $\eta=t-1$ is

$$
-\frac{\Gamma(1-t)}{\Gamma(s)} \Gamma(s+t-1) \zeta(s+t-1)
$$


unless $t=1,2,3, \ldots$, we shift the contour to obtain

$$
h(s, t)=\frac{1}{2 \pi i} \int_{C} \frac{\Gamma(s+\eta) \Gamma(-\eta)}{\Gamma(s)} \zeta(t-\eta) \zeta(s+\eta) d \eta
$$

for $s, t \in \mathbb{C}$ with $s \neq 1-k$ and $t \neq k(k=0,1,2, \ldots)$, where the contour $C$ is a line from $-i \infty$ to $i \infty$ indented in such a manner as to separate the points at $\eta=-s+1-l, t-l(l=0,1,2, \ldots)$ from the points at $\eta=0,1,2, \ldots$ By the functional equation of the Riemann zeta function, the integrand is equal to $(2 \pi)^{s+t-1} \Gamma(1-t)$ times

$$
\begin{aligned}
& \cos \left(\frac{\pi}{2}(s+t-1)\right) \frac{\Gamma(1-t+\eta) \Gamma(-\eta)}{\Gamma(s) \Gamma(1-t)} \zeta(1-s-\eta) \zeta(1-t+\eta) \\
& +\sin \left(\frac{\pi}{2}(s+t-1)\right) \frac{\Gamma(1-s)}{\pi} \sin (\pi(1-s)) \\
& \quad \times \cot \left(\frac{\pi}{2}(1-s-\eta)\right) \frac{\Gamma(1-t+\eta) \Gamma(-\eta)}{\Gamma(1-t)} \zeta(1-s-\eta) \zeta(1-t+\eta) .
\end{aligned}
$$

Thus, we obtain (A.1).

We now compare our result with the result of Matsumoto to obtain a new representation of $A(s, t ; 0)$. For $(s, t) \in \mathbb{C}^{2}$ with $\operatorname{Re}(s)<0$ and $\operatorname{Re}(t)>1$, set

$$
F_{ \pm}(s, t)=\sum_{k=1}^{\infty} \sigma_{s+t-1}(k) \Psi(t, s+t ; \pm 2 \pi i k),
$$

where $\sigma_{\nu}(k)=\sum_{d \mid k} d^{\nu}$ and $\Psi(\alpha, \gamma ; z)$ is the confluent hypergeometric function of the second kind. It is known that $F_{ \pm}(s, t)$ can be continued meromorphically to the whole space $\mathbb{C}^{2}$.

Corollary A.1. The function $A(s, t ; 0)$ can be represented in terms of the $F_{ \pm}$-functions:

$$
2 \Gamma(s) A(s, t ; 0)=(2 \pi i)^{s+t} F_{+}(s, t)+(-2 \pi i)^{s+t} F_{-}(s, t) .
$$

Proof. Propositions 1 and 2 in [8] show

$$
\frac{h(s, t)}{(2 \pi)^{s+t-1} \Gamma(1-t)}=e^{\pi i(s+t-1) / 2} F_{+}(t, s)+e^{\pi i(1-s-t) / 2} F_{-}(t, s)
$$

and

$$
F_{ \pm}(1-t, 1-s)=( \pm 2 \pi i)^{s+t-1} F_{ \pm}(s, t),
$$

respectively. These suggest

$$
\frac{h(1-t, 1-s)}{\Gamma(s)}=F_{+}(t, s)+F_{-}(t, s)
$$

and so we see

$$
\begin{aligned}
\frac{h(s, t)}{(2 \pi)^{s+t-1} \Gamma(1-t)}= & \cos \left(\frac{\pi}{2}(s+t-1)\right) \frac{h(1-t, 1-s)}{\Gamma(s)} \\
& +i \sin \left(\frac{\pi}{2}(s+t-1)\right)\left(F_{+}(t, s)-F_{-}(t, s)\right) .
\end{aligned}
$$

By comparing this with (A.1), we have $\Gamma(1-s) A(1-s, 1-t ; 0)=\pi i\left(F_{+}(t, s)-F_{-}(t, s)\right)$. Thus, we use (A.2) to obtain the result. 


\section{REFERENCES}

[1] W. Chu and C. Wang, Convolution formulae for Bernoulli numbers, Integral Transforms Spec. Funct. 21 (2010), 437-457.

[2] J. G. Huard, K. S. Williams and N.-Y. Zhang, On Tornheim's double series, Acta Arith. 75 (1996), $105-117$.

[3] Y. Komori, An integral representation of the Mordell-Tornheim double zeta function and its values at non-positive integers, Ramanujan J. 17 (2008), 163-183.

[4] Y. Komori, K. Matsumoto and H. Tsumura, Functional equations and functional relations for the Euler double zeta-function and its generalization of Eisenstein type, Publ. Math. Debrecen 77 (2010), 15-31.

[5] N. Kurokawa and H. Ochiai, Zeros of Witten zeta functions and applications, preprint 2011.

[6] Z. Li, On functional relations for the alternating analogues of Tornheim's double zeta function, arXiv:1011.2897 1 .

[7] K. Matsumoto, On the analytic continuation of various multiple zeta-functions, in: Number Theory for the Millennium II, Proc. Millennial Conference on Number Theory, Urbana-Champaign, 2000, (eds. M. A. Bennett et al.), A. K. Peters, 2002, 417-440.

[8] K. Matsumoto, Functional equations for double zeta-functions, Math. Proc. Camb. Phil. Soc. 136 (2004), $1-7$.

[9] T. Nakamura, A functional relation for the Tornheim double zeta function, Acta Arith. 125 (2006), $257-263$.

[10] K. Onodera, Mordell-Tornheim multiple zeta values at non-positive integers, to appear in Ramanujan J.

[11] R. Sczech, Eisenstein cocycles for $G L_{2} \mathbb{Q}$ and values of $L$-functions in real quadratic fields, Comment. Math. Helv. 67 (1992), 363-382.

[12] L. Tornheim, Harmonic double series, Amer. J. Math. 72 (1950), 303-314.

[13] H. Tsumura, On functional relations between the Mordell-Tornheim double zeta functions and the Riemann zeta function, Math. Proc. Cambridge Philos. Soc. 142 (2007), 395-405.

[14] A. Weil, Elliptic functions according to Eisenstein and Kronecker, Springer-Verlag, Berlin, Heidelberg, New York, 1976.

[15] E. T. Whittaker and G. N. Watson, A course of modern analysis, 4th ed., Cambridge Univ. Press, 1927.

Department of Mathematics, Tokyo Institute of Technology, O-okayama, Meguro-Ku, Tokyo 152-8551, JAPAN

E-mail address: onodera@math.titech.ac.jp 